\title{
Prognostic value of pulmonary vein size in prediction of atrial fibrillation recurrence after pulmonary vein isolation: a cardiovascular magnetic resonance study
}

\author{
Thomas H. Hauser ${ }^{1 *} \mathbb{D}$, Vidal Essebag ${ }^{2}$, Ferdinando Baldessin³ ${ }^{3}$ Seth McClennen ${ }^{4}$, Susan B. Yeon,
} Warren J. Manning ${ }^{1,6}$ and Mark E. Josephson ${ }^{1}$

\begin{abstract}
Background: The relationship between pulmonary vein (PV) anatomy and successful catheter ablation of atrial fibrillation (AF) is poorly understood

Methods: First-pass contrast enhanced PV magnetic resonance angiography was performed in 71 consecutive patients prior to PV isolation. PV diameter and cross-sectional area (CSA) were measured prior to PV isolation. Any symptomatic or asymptomatic $A F>10$ s was considered a recurrence. Early recurrence was defined as recurrent $A F$ $\leq 30$ days after PV isolation, while late recurrence of AF was defined as recurrent AF $>30$ days after.

Results: At 1 year, $57 \%$ had any recurrence of AF while $41 \%$ had late recurrence of AF. Study subjects with one or more PV diameter in the top $10^{\text {th }}$ percentile had trend toward more early recurrent AF (HR 1.99, $\left.p=0.053\right)$. Study subjects with one or more PV CSA in the top $10^{\text {th }}$ percentile had more late recurrent AF (HR 2.25, $\left.p=0.039\right)$ and a trend toward more early recurrent AF (HR 1.94, $p=0.064)$. With multivariate analysis, PV size was not associated with early recurrent AF, but late recurrent AF was associated with one or more large PV, increased left atrial size, and non-paroxysmal AF. Study subjects with all three of these risk factors had a $100 \%$ rate of late recurrent AF at 1 year, while those with none had a $7 \%$ rate of late recurrent AF.
\end{abstract}

Conclusions: Larger PV size is independently associated with more late recurrent AF after PV isolation. Determination of PV size prior to PV isolation may predict procedural success.

Keywords: Atrial fibrillation, Catheter ablation, Cardiovascular magnetic resonance, Pulmonary vein

\section{Background}

Atrial fibrillation (AF) is the most common sustained cardiac arrhythmia [1]. The recognition that the pulmonary veins (PV) have a critical role in the development and maintenance of AF has led to the development of several procedures to electrically isolate the PV from the left atrium to prevent recurrent AF [2-7]. The cause of recurrent $\mathrm{AF}$ is incompletely understood but is associated with electrical reconnection of the PV with the left atrium

\footnotetext{
* Correspondence: thauser@bidmc.harvard.edu

'Cardiovascular Division, Department of Medicine, Beth Israel Deaconess Medical Center and Harvard Medical School, 330 Brookline Avenue, RW-453, Boston 02215, Massachusetts

Full list of author information is available at the end of the article
}

$[8,9]$. The extent to which anatomic measurements of the PV might predict electrical reconnection has not been investigated.

We hypothesized that patients with large PV would be at higher risk for electrical reconnection and thus more recurrent $\mathrm{AF}$ after $\mathrm{PV}$ isolation. We evaluated this hypothesis in a consecutive series of 71 patients who underwent PV magnetic resonance angiography (MRA) prior to PV isolation.

\section{Methods}

\section{Study cohort}

The study cohort was comprised of a consecutive series of 71 patients who underwent cardiovascular magnetic 
resonance (CMR) prior to PV isolation for the treatment of AF. The clinical characteristics of the study cohort are summarized in Table 1. Antiarrhythmic drug therapy had failed in 58 (72\%) after the use of 1 (29 [41\%]), 2 (19 [27\%]), or 3 or more (10 [14 \%]) drugs. The study was approved by the hospital Committee on Clinical Investigations (Institutional Review Board), with waiver of written informed consent.

\section{CMR Technique}

CMR was performed using a $1.5 \mathrm{~T}$ whole-body MR system (Intera, Philips Medical Systems, Best, The Netherlands) with a five-element cardiac synergy coil for radiofrequency signal reception.

First pass breath-hold 3D contrast enhanced MRA of the PV was obtained after manual bolus administration of $0.2 \mathrm{mmol} / \mathrm{kg}$ gadopentetate dimeglumine (Magnevist ${ }^{\circ}$, Berlex Laboratories, Wayne, NJ), immediately followed by a saline flush. Data acquisition began after a delay determined by a small timing bolus given prior to contrast enhanced MRA. A spoiled endexpiratory breath-hold 3D gradient echo sequence with the following parameters was used: repetition time $3.6 \mathrm{~ms}$, echo time $1.1 \mathrm{~ms}$, flip angle 30 degrees, 50 slices, slice thickness $4 \mathrm{~mm}$ interpolated to $2 \mathrm{~mm}$, field of view $480 \mathrm{~mm}$, matrix $272 \times 512$, imaging time $22 \mathrm{~s}$. The 3D volume was centered on the left atrium and included all PV. Images were prospectively acquired in the axial plane. Commercially available system software (EasyVision 5.1 or ViewForum R5.1, Philips Medical Systems, Best, The Netherlands) was used to generate multiplanar reformations.

Steady-state free-precession breath-hold gradient echo ECG-gated cine CMR was performed in the 4-chamber and contiguous short axis orientations during a series of end-tidal breath-holds. Short axis images were acquired from the left ventricular base to the apex with $10 \mathrm{~mm}$ slices and no gap. The following parameters were used: repetition time $3.0 \mathrm{~ms}$, echo time $1.5 \mathrm{~ms}$, flip angle $60^{\circ}$, field of view $480 \mathrm{~mm}$, matrix $208 \times 256$.

Table 1 Characteristics of the study cohort and hazard ratios for the recurrence of a trial fibrillation (AF)

\begin{tabular}{|c|c|c|c|c|c|}
\hline \multirow[b]{2}{*}{ Characteristic } & & \multicolumn{2}{|l|}{ Early Recurrent AF } & \multicolumn{2}{|l|}{ Late Recurrent AF } \\
\hline & & HR (95 \%) & $p$ & HR $(95 \% \mathrm{Cl})$ & $p$ \\
\hline \multicolumn{6}{|l|}{ Demographics } \\
\hline Men & $55(77 \%)$ & $3.62(1.10-11.88)$ & 0.034 & $1.84(0.64-5.32)$ & 0.261 \\
\hline Age, years * & $52 \pm 11$ & $1.27(0.92-1.75)$ & 0.147 & $1.01(0.97-1.05)$ & 0.621 \\
\hline Body surface area, $\mathrm{m}^{2}$ & $2.10 \pm 0.22$ & $0.68(0.14-3.39)$ & 0.634 & $0.36(0.07-1.97)$ & 0.237 \\
\hline Body mass index, $\mathrm{kg} / \mathrm{m}^{2}$ & $29.3 \pm 5.6$ & $0.99(0.93-1.06)$ & 0.762 & $0.99(0.92-1.06)$ & 0.780 \\
\hline \multicolumn{6}{|l|}{ Type of atrial fibrillation } \\
\hline Paroxysmal & $40(56 \%)$ & $0.36(0.18-0.73)$ & 0.004 & $0.36(0.17-0.80)$ & 0.012 \\
\hline Persistent & $25(35 \%)$ & & & & \\
\hline Permanent & $6(8 \%)$ & & & & \\
\hline \multicolumn{6}{|l|}{ Medical history } \\
\hline Mitral regurgitation † & $33(46 \%)$ & $1.68(0.85-3.35)$ & 0.138 & $1.52(0.71-3.25)$ & 0.280 \\
\hline Hypertension & $32(45 \%)$ & $1.27(0.64-2.52)$ & 0.488 & $1.38(0.64-2.95)$ & 0.409 \\
\hline Obstructive sleep apnea & $19(27 \%)$ & $0.79(0.35-1.74)$ & 0.551 & $1.17(0.51-2.68)$ & 0.713 \\
\hline Diabetes mellitus & $10(14 \%)$ & $1.38(0.57-3.36)$ & 0.471 & $2.04(0.86-4.83)$ & 0.106 \\
\hline Coronary artery disease & $7(10 \%)$ & $1.27(0.44-3.61)$ & 0.659 & $1.54(0.46-5.15)$ & 0.489 \\
\hline Obstructive lung disease & $3(4 \%)$ & 0 (not defined) $\|$ & 0.991 & 0 (not defined) $\|$ & 0.991 \\
\hline No comorbidity & $22(31 \%)$ & $0.71(0.32-1.58)$ & 0.400 & $0.89(0.38-2.12)$ & 0.799 \\
\hline \multicolumn{6}{|l|}{ CMR measurements } \\
\hline LV ejection fraction, \% * & $61 \pm 10$ & $0.92(0.66-1.28)$ & 0.609 & $1.22(0.79-1.88)$ & 0.371 \\
\hline LV end diastolic volume, $\mathrm{ml} *$ & $163 \pm 40$ & $0.91(0.82-1.01)$ & 0.067 & $0.92(0.82-1.04)$ & 0.168 \\
\hline LV mass, $g$ * & $129 \pm 34$ & $1.03(0.93-1.14)$ & 0.611 & $0.96(0.86-1.08)$ & 0.516 \\
\hline Left atrial dimension (4-chamber), $\mathrm{cm}$ & $5.7 \pm 0.8$ & $1.58(0.99-2.51)$ & 0.056 & $2.20(1.38-3.49)$ & $<0.001$ \\
\hline
\end{tabular}

* The hazard ratio is reported for a 10 unit change

† Determined by the presence of $\geq$ mild mitral regurgitation on echocardiography

II No subjects with obstructive lung disease had a recurrence of $A F$

$\mathrm{HR}=$ hazard ratio, $\mathrm{Cl}=$ confidence interval, $\mathrm{LV}=$ left ventricular 


\section{Image Analysis}

All measurements were made by an observer blinded to the clinical treatment data using commercially available system software (EasyVision 5.1, Philips Medical Systems, Best, The Netherlands). The maximum left atrial dimension was measured in the 4-chamber orientation. Left ventricular volumes, mass and ejection fraction were determined using Simpson's rule [10]. The PV were measured at the location in the sagittal plane at which the PV separate from the left atrium and from each other identified by viewing a sagittal plane reconstruction of the dataset [11]. The maximal diameter and cross-sectional area (CSA) of each PV were measured. A left common PV was defined as a single left-sided PV entering the left atrium as determined in the sagittal plane. A right middle PV was defined as any right-sided pulmonary vein identified in the sagittal plane in addition to the right inferior and right superior PV.

Any PV with a diameter in the top $10^{\text {th }}$ percentile of all PV was defined as having a large diameter. Similarly, any PV with a CSA in the top $10^{\text {th }}$ percentile was defined as having a large CSA. A separate analysis was performed for each measure of PV size.

\section{PV Isolation Procedure}

The electrophysiology procedure was performed using a femoral venous approach. A decapolar catheter was positioned in the coronary sinus and a second catheter was placed in the right atrium. Left atrial access was obtained by two transseptal punctures. Transesophageal or intracardiac echocardiography was used to identify PV anatomy and velocities before and after ablation, to identify catheter position with reference to the vein ostia, to determine the presence or absence of pericardial effusion, and in some cases to guide transseptal puncture. Following transseptal puncture, patients received intravenous heparin to maintain a serum activated clotting time $>250 \mathrm{~s}$.

Three-dimensional electroanatomic mapping of the left atrium and PV was performed using a non-irrigated 4 or $8 \mathrm{~mm}$ tip NaviStar ${ }^{\mathrm{Tm}}$ catheter (Biosense Webster) and CARTO $^{\text {mix }}$ (Biosense Webster) and/or EnSite NavX ${ }^{\mathrm{ma}}$ (Endocardial Solutions) recording systems. Electrograms were recorded during sinus rhythm, coronary sinus pacing, or AF at the ostia of the PV with a 10-14 pole circumferential catheter with distal ring configuration (Biosense Webster or Bard). Radiofrequency ablation was performed outside the PV ostium near sites with the earliest PV electrograms. Ablation was performed for 20-60 s with a target temperature of $52{ }^{\circ} \mathrm{C}$. Temperature was considered adequate if it reached $45^{\circ} \mathrm{C}$. The process was repeated until complete bi-directional electrical PV isolation was achieved, defined by both entrance block as demonstrated by loss of PV potentials, and exit block demonstrated by failure to capture the left atrium during sinus rhythm by pacing (at $10 \mathrm{~mA}$ and $2 \mathrm{~ms}$ pulse width) each of the bipolar pairs of electrodes of the circumferential catheter positioned at the entrance of the PV. All PV were routinely isolated for all patients.

After PV isolation, induction of AF was attempted by burst pacing from the right atrium and coronary sinus before and after administration of isoproterenol. Isolation of the PV was reassessed and if reconnection was observed the vein was re-isolated. Left atrial ablation lines (mitral isthmus line and/or posterior left atrial line) were performed in $16(23 \%)$ patients with inducible sustained left atrial tachycardia. A right atrial isthmus line (tricuspid valve to inferior vena cava) was performed in $17(24 \%)$ patients with a history of or inducible right atrial isthmus dependent flutter.

\section{Post ablation care and monitoring}

Patients were treated with warfarin to achieve an international normalized ratio of 2.0 to 3.0 for at least 6 months after PV isolation, as well as acetylsalicylic acid (325 mg/day) for at least 1 month. Antiarrhythmic drugs were continued post procedure for patients with a history of persistent or permanent AF, and were reinitiated in patients with early ( $<30$ days) recurrences of AF. Antiarrhythmic drugs were used in 29 (41 \%) patients post procedure, including 12 (29\%) free of AF at last follow-up.

Evaluation of symptomatic or asymptomatic AF was performed using both a 2 week transtelephonic event recorder and $24 \mathrm{~h}$ Holter monitor at 1, 3, 6, and 12 months. Additional monitoring was done for patients with symptoms. Recurrent AF was deemed present if an asymptomatic or symptomatic atrial tachyarrhythmia consistent with AF was documented to last $>10 \mathrm{~s}$. Early recurrent $\mathrm{AF}$ was defined as any recurrent $\mathrm{AF}$ during the first 30 days. Late recurrent AF was defined as recurrent AF after 30 days. The median duration of follow-up was 442 days.

\section{Statistical Methods}

Continuous values are reported as the mean \pm standard deviation. Categorical values are reported as counts and percentages. The occurrence of recurrent AF was estimated using the product-limit (Kaplan-Meier) method. The relationship between PV size and recurrent AF was assessed using the product-limit method and the log rank test. The relationship of PV size and clinical variables with recurrent AF was assessed using proportional hazards regression. The proportional hazards assumption was confirmed for all variables by examining log-log survival curves. Multivariate analysis was performed using proportional hazards regression with backward selection of variables. Any variable with a $\mathrm{p}$-value of $\leq 0.1$ 
was eligible for inclusion and retention in the model. Hazard ratios (HR) are reported as the estimate with the $95 \%$ confidence interval (CI). A p-value of $<0.05$ was used for determination of statistical significance. All statistical analysis was performed using SAS for Windows (v9.3, SAS Institute, Cary, NC).

\section{Results}

The mean duration of the electrophysiological procedure was $257 \pm 63 \mathrm{~min}$. The mean number of ablations performed was $73 \pm 25$ with a mean total ablation time of $53 \pm 23 \mathrm{~min}$. There was no relationship between the procedure time, total number of ablations or total ablation time with any measure of PV size or with the presence of one or more large PV by any measurement criteria ( $\mathrm{p}>0.15$ for all).

At 1 year of follow-up, $57 \%$ had any recurrent $\mathrm{AF}$ and $41 \%$ had late recurrence of AF (Fig. 1). The relationship of the clinical characteristics of the cohort with the recurrence of AF are shown in Table 1. There was no relationship of the performance of additional left or right atrial ablation lines with the recurrence of AF ( $\mathrm{p}>0.7$ for both). Early recurrent AF was strongly associated with late recurrent AF (HR 5.14 (95 \% CI 2.06 - 12.80, $\mathrm{p}<0.001)$.

Size measurements for all PV are shown in Table 2. Data regarding the prognostic significance of PV size is shown in Table 3. The relationship of PV size to recurrent $\mathrm{AF}$ was examined by evaluating the recurrence in study subjects with one or more large PV as defined by boundary values at the $95^{\text {th }}, 90^{\text {th }}$, and $85^{\text {th }}$ percentiles of the individual PV measurements. The presence of one or more large PV CSA was associated with both early and late recurrence of $\mathrm{AF}$, while the presence of one or more large PV diameter was associated with early

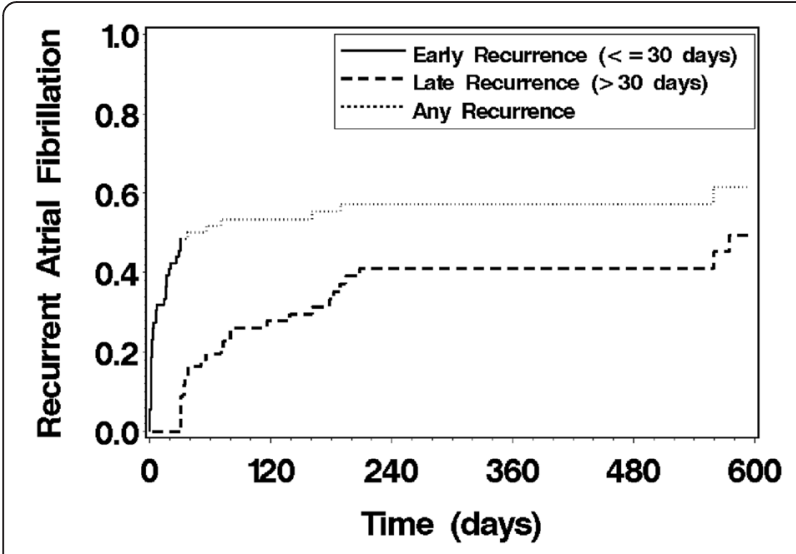

Fig. 1 Product-limit estimates for the probability of recurrent atrial fibrillation (AF) for the entire cohort, shown for those with early recurrent $A F$ (recurrence within the first 30 days), late recurrent $A F$ (recurrence after 30 days), or any recurrent AF
Table 2 Pulmonary vein measurements

\begin{tabular}{lccc}
\hline Pulmonary Vein & $\mathrm{N}$ & Diameter $(\mathrm{cm})$ & $\mathrm{CSA}\left(\mathrm{cm}^{2}\right)$ \\
\hline Left inferior & 58 & $1.8 \pm 0.4$ & $1.7 \pm 0.7$ \\
Left superior & 58 & $1.7 \pm 0.3$ & $1.7 \pm 0.5$ \\
Left common & 13 & $2.9 \pm 0.7$ & $3.4 \pm 1.1$ \\
Right inferior & 71 & $1.8 \pm 0.5$ & $2.3 \pm 1.0$ \\
Right middle & 7 & $0.9 \pm 0.1$ & $0.9 \pm 0.2$ \\
Right superior & 71 & $2.1 \pm 0.6$ & $2.9 \pm 1.3$ \\
All & 278 & $1.9 \pm 0.6$ & $2.2 \pm 1.1$ \\
\hline
\end{tabular}

CSA $=$ cross-sectional area

recurrence of AF only. The optimal boundary value for the diameter was the $90^{\text {th }}$ percentile. The $95^{\text {th }}$ and $90^{\text {th }}$ percentile boundary values for the CSA produced similar results for late recurrence of AF. Based on these results, we chose a boundary value of the $90^{\text {th }}$ percentile as the optimal measure to determine that a PV was large. Using this criterion, 24 (34\%) patients had one or more PV with a large diameter and 23 (32\%) had one or more PV with a large CSA. Multiple large PV were present in 2 patients with large diameter PV and 3 patients with large CSA PV. The distribution of large PV is shown in Table 4. Left common and right superior PV were most likely to have increased size while no left superior or right middle PV were large.

The relationship between recurrent AF and the presence of one or more large CSA PV was further investigated with multivariate proportional hazards regression (Table 5). With simultaneous adjustment for male gender, the presence of paroxysmal AF, and left ventricular (LV) end diastolic volume, left atrial dimension and one or more large PV CSA were no longer significantly related to early recurrence of $\mathrm{AF}$ and were removed from the model. In the analysis of late recurrence of AF, paroxysmal AF, left atrial dimension, and one or more large PV CSA remained in the model after simultaneous adjustment for each the effects of each of these variables. Adjustment for age and gender did not significantly affect these results.

We further evaluated the late recurrence of AF by stratification of study subjects by the presence of one or more large PV by CSA criteria (Fig. 2), which showed a significant difference between the groups $(\mathrm{p}=0.032)$. Multivariate proportional hazards regression identified 3 significant risk factors for late recurrent AF: nonparoxysmal AF, increasing left atrial dimension, and the presence of one or more large PV CSA. Stratification of the study cohort by the number of these risk factors present identified three risk groups for recurrence (Fig. 3). Those study subjects with all 3 risk factors had a high rate of recurrence (100 \% by 1 year). Those study subjects with no risk factors had a very low recurrence 
Table 3 Prognostic value of pulmonary vein size for the prediction of recurrent atrial fibrillation

\begin{tabular}{|c|c|c|c|c|c|c|}
\hline & & & Early Recurrent AF & & Late Recurrent AF & \\
\hline Pulmonary Vein Measure & Boundary* & $\mathrm{N}+$ & HR (95 \%) & $\mathrm{p}$ & $\mathrm{HR}(95 \% \mathrm{Cl})$ & $\mathrm{p}$ \\
\hline \multicolumn{7}{|l|}{ Diameter } \\
\hline $95^{\text {th }}$ percentile & $3.0 \mathrm{~cm}$ & $8(11 \%)$ & $1.88(0.77-4.56)$ & 0.165 & $1.28(0.44-3.70)$ & 0.653 \\
\hline $90^{\text {th }}$ percentile & $2.7 \mathrm{~cm}$ & $21(30 \%)$ & $1.98(0.99-3.96)$ & 0.053 & $1.47(0.67-3.21)$ & 0.335 \\
\hline $85^{\text {th }}$ percentile & $2.4 \mathrm{~cm}$ & $31(44 \%)$ & $1.87(0.94-3.75)$ & 0.075 & $1.33(0.62-2.85)$ & 0.464 \\
\hline \multicolumn{7}{|l|}{ Cross-sectional area } \\
\hline $95^{\text {th }}$ percentile & $4.61 \mathrm{~cm}^{2}$ & $12(17 \%)$ & $2.28(1.05-4.95)$ & 0.037 & $2.51(1.05-6.00)$ & 0.039 \\
\hline $90^{\text {th }}$ percentile & $3.71 \mathrm{~cm}^{2}$ & $20(28 \%)$ & $1.94(0.96-3.91)$ & 0.064 & $2.25(1.04-4.88)$ & 0.039 \\
\hline $85^{\text {th }}$ percentile & $3.25 \mathrm{~cm}^{2}$ & $30(42 \%)$ & $1.52(0.77-3.01)$ & 0.232 & $1.98(0.92-4.24)$ & 0.079 \\
\hline
\end{tabular}

*The boundary value marks the percentile boundary for all pulmonary veins individually

$+\mathrm{N}$ represents the number of study subjects with at least one pulmonary vein measured $\geq$ boundary value

rate $(7 \%$ at 1 year). Study subjects with 1 or 2 risk factors had intermediate recurrence rates.

\section{Discussion}

In this prospective study of 71 consecutive patients undergoing CMR prior to PV isolation, we found that PV size, left atrial size, and paroxysmal AF are significantly and independently related to late recurrent AF after PV isolation. As PV isolation for the treatment of AF becomes more common, the appropriate selection of patients for these procedures becomes more important. In our study, $100 \%$ of study subjects with nonparoxysmal AF, increased left atrial size, and one or more PV with a CSA in the top $10^{\text {th }}$ percentile had recurrent AF at 1 year while only $7 \%$ of study subjects without these risk factors had late recurrent $\mathrm{AF}$ at 1 year.

The PV have a critical role in the pathophysiology of AF. The PV and left atrium are both derived from the primitive common PV [12] and therefore have many anatomic and histologic similarities. Both are smooth-walled structures that have electrically active myocardium. Approximately $90 \%$ of PVs contain atrial myocardium.[13] Although the myocardium in the atrium is uniform, myocardium in the PV is frequently discontinuous and fibrotic. Patients with a history of AF uniformly have myocardium in the PV and an

Table 4 Distribution of large pulmonary veins

\begin{tabular}{lrll}
\hline Pulmonary Vein & $N$ & Large Diameter $(\geq 2.7 \mathrm{~cm})$ & Large CSA $\left(\geq 3.71 \mathrm{~cm}^{2}\right)$ \\
\hline Left inferior & 58 & $1(2 \%)$ & $2(3 \%)$ \\
Left superior & 58 & $0(0 \%)$ & $0(0 \%)$ \\
Left common & 13 & $6(46 \%)$ & $5(38 \%)$ \\
Right inferior & 71 & $4(6 \%)$ & $5(7 \%)$ \\
Right middle & 7 & $0(0 \%)$ & $0(0 \%)$ \\
Right superior & 71 & $17(24 \%)$ & $15(21 \%)$
\end{tabular}

Large pulmonary veins (PV) were defined as PV in the top $10^{\text {th }}$ percentile for each measure of PV size. The percentage of large PV at each position compared to the total number of PV at that position is reported. $\mathrm{CSA}=$ cross-sectional area increased rate of structural abnormalities. These structural abnormalities result in abnormal electrical activation with slow and anisotropic conduction that result in proarrhythmic activity [14] that is directly responsible for the generation of AF in many patients [2]. Several catheter-based ablation procedures have been developed to electrically isolate the PV from the left atrium for the prevention of $\mathrm{AF}$ with short-term success rates ranging from 65 to $85 \%$ in patients with paroxysmal AF [2-7].

Recurrent AF after these procedures has generally been attributed to recovery of electrical function after the procedure.[8, 9] Larger PV may be at greater risk for electrical reconnection for three reasons. First, larger PV may be more difficult to isolate. Second, a larger perimeter may provide more opportunity for electrical reconnection to take place. Finally, larger PV may have a higher rate of histological and electrophysiological abnormalities that predispose to electrical reconnection. For these reasons, we hypothesized that patients with large PV would be at higher risk for electrical reconnection and thus more recurrent AF after PV isolation. We found that patients with one or more PV in the top $10^{\text {th }}$ percentile for CSA were at increased risk of late recurrent AF.

Paroxysmal AF has been previously described as predicting better outcome after PV isolation compared to persistent or permanent AF. [15-19] We also found that non-paroxysmal AF is independently associated with more recurrent AF. This may be due to left atrial remodeling due to permanent or persistent AF that may produce additional triggers for AF in addition to the PV.

Left atrial size has been previously reported to predict increased rates of recurrent $\mathrm{AF}$, independent of other predictors, [7, 20, 21] a finding that was confirmed in our cohort. A prior study found that left atrial size was a more closely correlated with recurrent AF independent of PV size [21]. We found that both left atrial size and PV size were independently associated with late recurrent AF. This difference may be due to different methods 
Table 5 Multivariate proportional hazards regression for the recurrence of atrial fibrillation

\begin{tabular}{|c|c|c|c|c|c|c|}
\hline & \multicolumn{2}{|l|}{ First Iteration } & \multicolumn{2}{|l|}{ Second Iteration } & \multicolumn{2}{|l|}{ Third Iteration } \\
\hline & $\mathrm{HR}(95 \% \mathrm{Cl})$ & $\mathrm{p}$ & $\mathrm{HR}(95 \% \mathrm{Cl})$ & $p$ & $\mathrm{HR}(95 \% \mathrm{Cl})$ & $\mathrm{p}$ \\
\hline \multicolumn{7}{|l|}{ Early recurrent AF } \\
\hline Men & $3.31(0.93-11.8)$ & 0.065 & $3.35(0.94-11.97)$ & 0.063 & $4.06(1.17-14.04)$ & 0.027 \\
\hline Paroxysmal AF & $0.47(0.22-1.00)$ & 0.049 & $0.44(0.21-0.90)$ & 0.025 & $0.46(0.23-0.95)$ & 0.036 \\
\hline LV end diastolic volume* & $0.87(0.77-0.97)$ & 0.014 & $0.86(0.77-0.97)$ & 0.011 & $0.86(0.77-0.97)$ & 0.014 \\
\hline Left atrial dimension & $1.14(0.73-1.76)$ & 0.573 & Removed & & Removed & \\
\hline One or more large PV CSA & $1.72(0.83-3.60)$ & 0.148 & $1.79(0.86-3.69)$ & 0.118 & Removed & \\
\hline \multicolumn{7}{|l|}{ Late recurrent AF } \\
\hline Paroxysmal AF & $0.47(0.20-1.11)$ & 0.086 & & & & \\
\hline Left atrial dimension & $1.78(1.08-2.93)$ & 0.023 & & & & \\
\hline One or more large PV CSA & $2.07(0.95-4.52)$ & 0.069 & & & & \\
\hline
\end{tabular}

$\mathrm{AF}=$ atrial fibrillation, $\mathrm{Cl}=$ confidence interval, $\mathrm{CSA}=$ cross-sectional area, $\mathrm{HR}=$ hazard ratio, $\mathrm{LV}=$ left ventricle, $\mathrm{PV}=$ pulmonary vein .

* The hazard ratio is reported for a 10 unit change

used to measure of left atrial and PV size in the prior report.

Left atrial fibrosis has also been described as a predictor of recurrent AF after PV isolation [22]. We did not routinely obtain pre-ablation late gadolinium enhanced images in this cohort. Prediction of recurrent AF based on the size of the PV appears to compare favorably to prediction based on fibrosis, but assessing both PV size and left atrial fibrosis in patients where both are measured would be required to provide an accurate comparison of predictive accuracy.

We found no other significant relationships of late recurrent AF with any other clinical or anatomic factors. Although advancing age [16], diabetes[23], and the presence of mitral regurgitation [19] have been previously reported

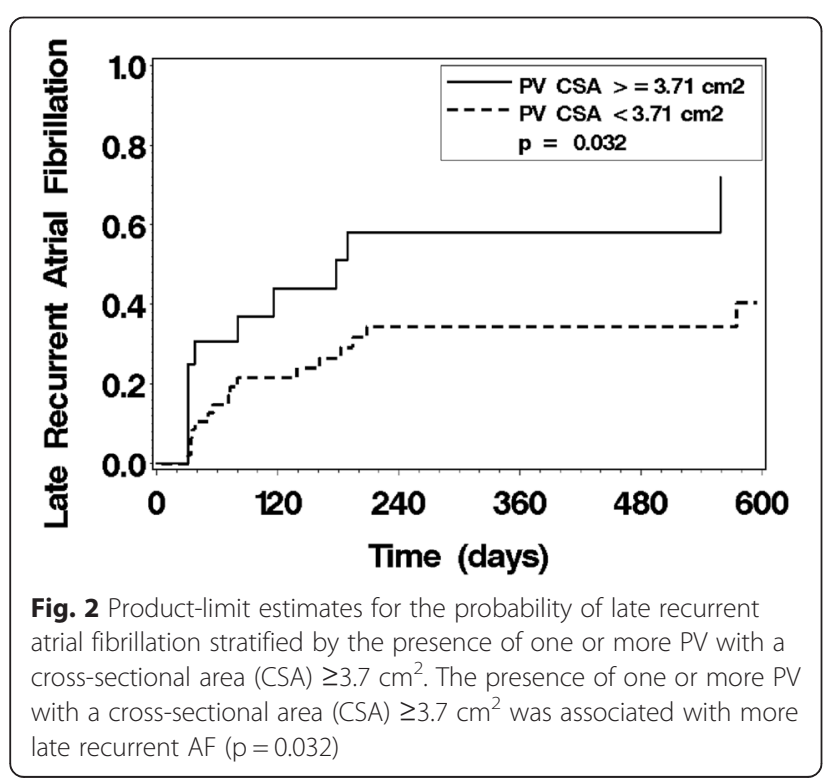

as associated with recurrent AF, we found no such relationships. This may be due to the relatively small sample size of this study.

We also examined early recurrence of AF in our dataset and found that male gender, paroxysmal AF, LV cavity size, left atrial size and the presence of one or more large PV were all associated with early recurrence of AF. With multivariate analysis, male gender, paroxysmal AF and LV cavity size remained significantly associated with early recurrence of AF. Male gender [24] and left atrial size [25] have previously been reported to have an association with early recurrent AF. Although prior studies have found a variable relationship of early recurrent $\mathrm{AF}$ with late recurrent AF [24-26], we found a significant relationship between the two. The variable findings may

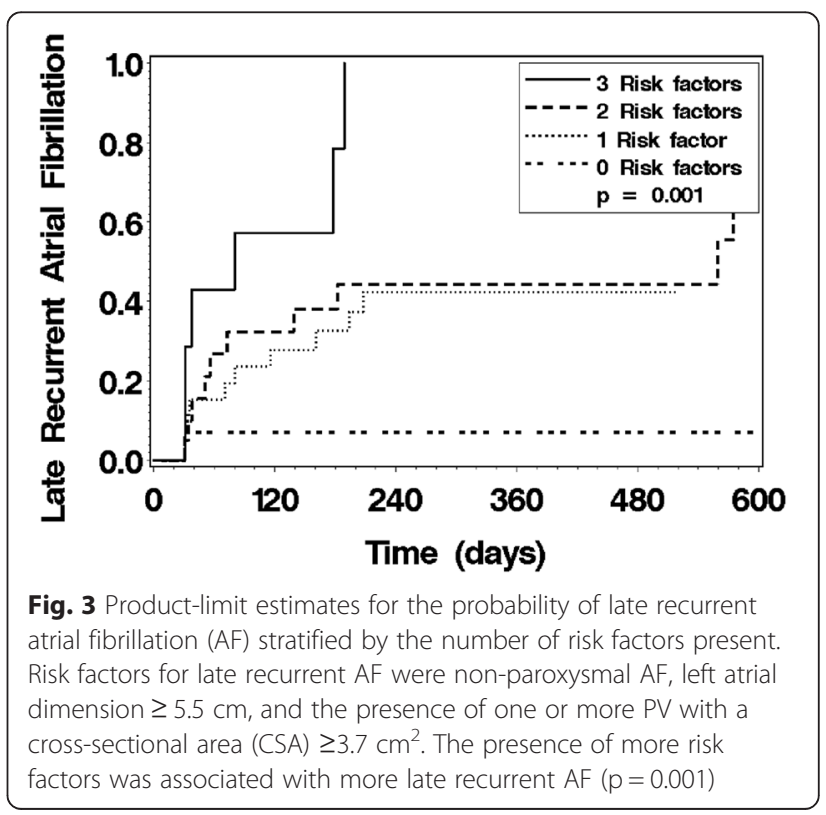


be due to different definitions of early compared to late recurrent AF.

We rigorously screened our study cohort for symptomatic or asymptomatic AF using both a 2 week transtelephonic event recorder and $24 \mathrm{~h}$ Holter monitor at each follow-up interval as well as additional monitoring for patients with symptoms. All patients with any recurrent $\mathrm{AF}$ were deemed a treatment failure even if sinus rhythm was later restored. As a result, our rate of late recurrent $\mathrm{AF}$ of $41 \%$ at 1 year is higher than that reported by other investigators [2-7].

\section{Conclusions}

Among patients referred for their initial PV isolation, the presence of one or more large PV CSA, non-paroxysmal $\mathrm{AF}$, and increased left atrial size were independent predictors of late recurrent $\mathrm{AF}$ after PV isolation. Stratification by the number of these factors present identified three risk groups. Patients with all three risk factors had a very high rate of recurrent AF. Patients with no risk factors had a very low rate of recurrent $\mathrm{AF}$. The remaining patients had an intermediate rate of recurrent AF. These results suggest that determination of PV size prior to PV isolation may predict procedural success and identify patients for whom the procedure is unlikely to result in long-term success.

\section{Abbreviations}

AF: atrial fibrillation; Cl: confidence interval; CMR: cardiovascular magnetic resonance; CSA: cross-sectional area; LV: left ventricle; MRA: magnetic resonance angiography; PV: pulmonary veinv.

\section{Competing interests}

The authors report no conflicts with regard to this work.

\section{Authors' contributions}

THH, WJM and MEJ conceived and designed the study. THH, VE, FB, SM, SBY, WJM, and MEJ acquired, analyzed and/or interpreted the data. THH, VE, WJM and MEJ drafted the manuscript. All authors reviewed, critically revised, and approved the manuscript. All authors read and approved the final manuscript.

\section{Acknowledgements}

Dr. Hauser was supported by the NIH loan repayment program. The authors greatly appreciate the careful review of the manuscript by Reza Nezafat, PhD.

\begin{abstract}
Author details
${ }^{1}$ Cardiovascular Division, Department of Medicine, Beth Israel Deaconess Medical Center and Harvard Medical School, 330 Brookline Avenue, RW-453, Boston 02215, Massachusetts. ${ }^{2}$ Division of Cardiology, McGill University Health Center, Montreal, Canada. ${ }^{3}$ Azienda Ospedaliera Treviso, Treviso, Italy. ${ }^{4}$ Harbor Medical Associates, South Weymouth, Massachusetts. ${ }^{5}$ UpToDate, Waltham, Massachusetts. ${ }^{6}$ Department of Radiology, Beth Israel Deaconess Medical Center, Boston, Massachusetts.
\end{abstract}

Received: 26 February 2015 Accepted: 1 June 2015 Published online: 18 June 2015

\section{References}

1. Feinberg WM, Blackshear JL, Laupacis A, Kronmal R, Hart RG. Prevalence, age distribution, and gender of patients with atrial fibrillation Analysis and implications. Arch Intern Med. 1995:155:469-73.

2. Haissaguerre $M$, Jais P, Shah DC, Takahashi A, Hocini M, Quiniou G, et al. Spontaneous initiation of atrial fibrillation by ectopic beats originating in the pulmonary veins. N Engl J Med. 1998;339:659-66.
3. Pappone C, Rosanio S, Oreto G, Tocchi M, Gugliotta F, Vicedomini G, et al. Circumferential radiofrequency ablation of pulmonary vein ostia. A new anatomic approach for curing atrial fibrillation. Circulation. 2000;102:2619-28.

4. Arentz T, von Rosenthal J, Blum T, Stockinger J, Burkle G, Weber R, et al. Feasibility and safety of pulmonary vein isolation using a new mapping and navigation system in patients with refractory atrial fibrillation. Circulation. 2003;108:2484-90.

5. Haissaguerre M, Jais P, Shah DC, Garrigue S, Takahashi A, Lavergne T, et al. Electrophysiological end point for catheter ablation of atrial fibrillation initiated from multiple pulmonary venous foci. Circulation. 2000;101:1409-17.

6. Oral H, Knight BP, Ozaydin M, Chugh A, Lai SW, Scharf C, et al. Segmental ostial ablation to isolate the pulmonary veins during atrial fibrillation: feasibility and mechanistic insights. Circulation. 2002;106:1256-62.

7. Pappone C, Rosanio S, Augello G, Gallus G, Vicedomini G, Mazzone P, et al. Mortality, morbidity, and quality of life after circumferential pulmonary vein ablation for atrial fibrillation: outcomes from a controlled nonrandomized long-term study. J Am Coll Cardiol. 2003;42:185-97.

8. Lemola K, Hall B, Cheung P, Good E, Han J, Tamirisa K, et al. Mechanisms of recurrent atrial fibrillation after pulmonary vein isolation by segmental ostial ablation. Heart Rhythm. 2004;1:197-202.

9. Ouyang F, Antz M, Ernst S, Hachiya H, Mavrakis H, Deger FT, et al. Recovered pulmonary vein conduction as a dominant factor for recurrent atrial tachyarrhythmias after complete circular isolation of the pulmonary veins: lessons from double Lasso technique. Circulation. 2005;111:127-35.

10. Salton CJ, Chuang ML, O'Donnell CJ, Kupka MJ, Larson MG, Kissinger KV, et al. Gender differences and normal left ventricular anatomy in an adult population free of hypertension. A cardiovascular magnetic resonance study of the Framingham Heart Study Offspring cohort. J Am Coll Cardiol. 2002;39:1055-60.

11. Hauser $T H$, Yeon SB, McClennen S, Katsimaglis G, Kissinger KV, Josephson $M E$, et al. A method for the determination of proximal pulmonary vein size using contrast-enhanced magnetic resonance angiography. J Cardiovasc Magn Reson. 2004;6:927-36.

12. Moore KL. The Developing Human. Philadelphia: Saunders; 1988.

13. Hassink RJ, Aretz HT, Ruskin J, Keane D. Morphology of atrial myocardium in human pulmonary veins: a postmortem analysis in patients with and without atrial fibrillation. J Am Coll Cardiol. 2003;42:1108-14.

14. Arora R, Verheule S, Scott L, Navarrete A, Katari V, Wilson E, et al. Arrhythmogenic substrate of the pulmonary veins assessed by high-resolution optical mapping. Circulation. 2003;107:1816-21.

15. Kojodjojo P, O'Neill MD, Lim PB, Malcolm-Lawes L, Whinnett ZI, Salukhe TV, et al. Pulmonary venous isolation by antral ablation with a large cryoballoon for treatment of paroxysmal and persistent atrial fibrillation: medium-term outcomes and non-randomised comparison with pulmonary venous isolation by radiofrequency ablation. Heart. 2010;96:1379-84.

16. Vasamreddy CR, Lickfett L, Jayam VK, Nasir K, Bradley DJ, Eldadah Z, et al. Predictors of recurrence following catheter ablation of atrial fibrillation using an irrigated-tip ablation catheter. J Cardiovasc Electrophysiol. 2004;15:692-7.

17. Berkowitsch A, Greiss H, Vukajlovic D, Kuniss M, Neumann T, Zaltsberg S, et al. Usefulness of atrial fibrillation burden as a predictor for success of pulmonary vein isolation. Pacing Clin Electrophysiol. 2005;28:1292-301.

18. Oral H, Scharf C, Chugh A, Hall B, Cheung P, Good E, et al. Catheter ablation for paroxysmal atrial fibrillation: segmental pulmonary vein ostial ablation versus left atrial ablation. Circulation. 2003;108:2355-60.

19. Yasuda T, Kumagai K, Ogawa M, Noguchi H, Tojo H, Matsumoto N, et al. Predictors of successful catheter ablation for atrial fibrillation using the pulmonary vein isolation technique. J Cardiol. 2004:44:53-8.

20. Helms AS, West JJ, Patel A, Lipinski MJ, Mangrum JM, Mounsey JP, et al. Relation of left atrial volume from three-dimensional computed tomography to atrial fibrillation recurrence following ablation. Am J Cardiol. 2009;103:989-93.

21. den Uijl DW, Tops LF, Delgado V, Schuijf JD, Kroft LJ, de Roos A, et al. Effect of pulmonary vein anatomy and left atrial dimensions on outcome of circumferential radiofrequency catheter ablation for atrial fibrillation. Am J Cardiol. 2011;107:243-9.

22. Marrouche NF, Wilber D, Hindricks G, Jais P, Akoum N, Marchlinski F, et al. Association of atrial tissue fibrosis identified by delayed enhancement MRI and atrial fibrillation catheter ablation: the DECAAF study. Jama. 2014;311:498-506. 
23. Wokhlu A, Hodge DO, Monahan KH, Asirvatham SJ, Friedman PA, Munger $\mathrm{TM}$, et al. Long-term outcome of atrial fibrillation ablation: impact and predictors of very late recurrence. J Cardiovasc Electrophysiol. 2010;21:1071-8.

24. Andrade JG, Khairy P, Macle L, Packer DL, Lehmann JW, Holcomb RG, et al. Incidence and significance of early recurrences of atrial fibrillation after cryoballoon ablation: insights from the multicenter Sustained Treatment of Paroxysmal Atrial Fibrillation (STOP AF) Trial. Circ Arrhythm Electrophysiol. 2014;7:69-75.

25. Jiang H, Lu Z, Lei H, Zhao D, Yang B, Huang C. Predictors of early recurrence and delayed cure after segmental pulmonary vein isolation for paroxysmal atrial fibrillation without structural heart disease. J Interv Card Electrophysiol. 2006;15:157-63.

26. Oral H, Knight BP, Ozaydin M, Tada H, Chugh A, Hassan S, et al. Clinical significance of early recurrences of atrial fibrillation after pulmonary vein isolation. J Am Coll Cardiol. 2002;40:100-4.

\section{Submit your next manuscript to BioMed Central and take full advantage of:}

- Convenient online submission

- Thorough peer review

- No space constraints or color figure charges

- Immediate publication on acceptance

- Inclusion in PubMed, CAS, Scopus and Google Scholar

- Research which is freely available for redistribution 